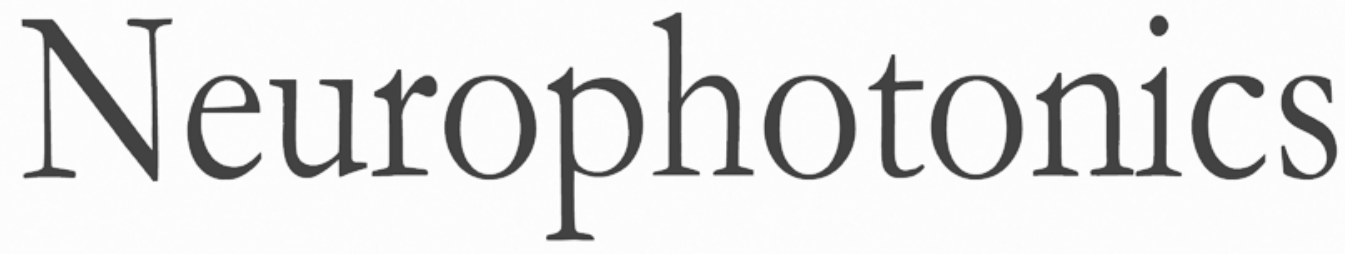

\title{
Imaging neural events in zebrafish larvae with linear structured illumination light sheet fluorescence microscopy
}

Yang Liu

Savannah Dale

Rebecca Ball

Ariel J. VanLeuven

Andrew Sornborger

James D. Lauderdale

Peter Kner 


\title{
Imaging neural events in zebrafish larvae with linear structured illumination light sheet fluorescence microscopy
}

\author{
Yang Liu, ${ }^{\text {a }}$ Savannah Dale, ${ }^{\mathrm{b}}$ Rebecca Ball, ${ }^{\mathrm{c}}$ Ariel J. VanLeuven, ${ }^{\mathrm{c}}$ Andrew Sornborger, ${ }^{\mathrm{d}}$ James D. Lauderdale, ${ }^{\mathrm{c}, \mathrm{e}}$ \\ and Peter Kner ${ }^{a, *}$ \\ aUniversity of Georgia, College of Engineering, Athens, Georgia, United States \\ ${ }^{\mathrm{b} C l e m s o n}$ University, Department of Bioengineering, Clemson, South Carolina, United States \\ 'University of Georgia, Department of Cellular Biology, Athens, Georgia, United States \\ 'Los Alamos National Laboratory, Information Sciences, CCS-3, Los Alamos, New Mexico, United States \\ eUniversity of Georgia, Neuroscience Division of the Biomedical Health Sciences Institute, Athens, Georgia, United States
}

\begin{abstract}
Light sheet fluorescence microscopy (LSFM) is a powerful tool for investigating model organisms including zebrafish. However, due to scattering and refractive index variations within the sample, the resulting image often suffers from low contrast. Structured illumination (SI) has been combined with scanned LSFM to remove out-of-focus and scattered light using square-law detection. Here, we demonstrate that the combination of LSFM with linear reconstruction SI can further increase resolution and contrast in the vertical and axial directions compared to the widely adopted root-mean square reconstruction method while using the same input images. We apply this approach to imaging neural activity in 7-day postfertilization zebrafish larvae. We imaged two-dimensional sections of the zebrafish central nervous system in two colors at an effective frame rate of 7 frames per second. ( The Authors. Published by SPIE under a Creative Commons Attribution 4.0 Unported License. Distribution or reproduction of this work in whole or in part requires full attribution of the original publication, including its DOI. [DOI: 10.1117/1.NPh.6.1.015009]
\end{abstract}

Keywords: microscopy; structured illumination; light sheet fluorescence microscopy; optical sectioning.

Paper 18065R received Oct. 24, 2018; accepted for publication Feb. 13, 2019; published online Mar. 5, 2019.

\section{Introduction}

A fundamental problem in biomedical science is understanding the three-dimensional (3-D) structure of the brain and other organs at the cellular level. Large, thick specimens and live model organisms, such as zebrafish, C. elegans, and fruit flies, are widely used for studying organ development, human diseases, and the nervous system. ${ }^{1,2}$ Light sheet fluorescence microscopy (LSFM), also known as selective plane illumination microscopy (SPIM), ${ }^{3}$ is a prevalent imaging method that has gained wide attention during the past decade. Distinct from the widely used epi-illumination widefield (WF) microscope or laser scanning confocal microscope, the planar illumination scheme of the LSFM allows researchers to study biological samples in three dimensions with significantly less light exposure and drastically lower illumination intensity, ${ }^{4}$ while still allowing for improved optical sectioning. What makes this possible is the sheet-like illumination of the sample at the focal plane of the detection objective lens, which greatly suppresses out-of-focus light. Additionally, because of its WF detection scheme, LSFM has a much higher temporal resolution over a large field-of-view (FoV) compared with laser point scanning techniques, such as confocal microscopy ${ }^{5}$ and two-photon microscopy. ${ }^{6}$

Several groups have demonstrated the superior, high temporal performance of light sheet systems in both two and three dimensions. ${ }^{7-10}$ Various configurations of LSFM have been developed and applied to different sizes of samples. These include single cell imaging, ${ }^{11-13}$ small multicellular samples, such as C. elegans, D. melanogaster, and zebrafish embryos, ${ }^{3,14,15}$ samples which are hundreds of microns in size, such as

*Address all correspondence to Peter Kner, E-mail: kner@engr.uga.edu
C. elegans ${ }^{16}$ and zebrafish larvae, ${ }^{7}$ and even samples up to a few centimeters in size, such as whole mouse embryos ${ }^{17}$ and human prostate tissue. ${ }^{18}$ Even though LSFM provides a high temporal resolution and excellence in optical sectioning, the resulting images are greatly affected by the thickness of the sample, especially when applied to large and semiopaque samples. Scattering introduces extra background light into the resulting image, which leads to a lower signal-to-noise ratio (SNR) and reduced contrast.

A number of methods have been proposed to address this issue, such as using the nondiffracting Bessel ${ }^{19-21}$ and Airy ${ }^{22,23}$ beams for excitation. Unfortunately, the side lobes of the Bessel beam also introduce extra out-of-focus light, which worsens the sectioning performance of the system. With Airy beams, a deconvolution process is required, and this approach requires the side lobes of the beam to remain in focus, limiting the detection to $0.4 \mathrm{NA}^{24}$ Using confocal slit detection (CSD) or twophoton excitation in conjunction with a Bessel or Airy beam can help minimize this problem. However, because these measures result in a higher light dose on the sample, they result in an increased rate of photo bleaching and increased risk of photodamage to the sample. ${ }^{24}$

An alternative approach is combining structured illumination microscopy (SIM) ${ }^{25}$ with LSFM. SIM was first employed in conventional WF fluorescence microscopy as an optical sectioning method, which works by projecting a sinusoidal illumination pattern onto the sample and reconstructing the optically sectioned image using three images with a phase difference of $2 \pi / 3{ }^{25}$ SIM has been combined with LSFM by replacing the light sheet created by a cylindrical lens with a scanned Gaussian or Bessel beam that can be modulated to create an SI pattern. $^{20,26-28}$ This approach is referred to as digitally scanned light sheet microscopy (DSLM). For optical sectioning, three 
sinusoidal patterns are most commonly combined using the root mean square (RMS) reconstruction algorithm. ${ }^{25}$ HiLo microscopy has also been combined with LSFM as an alternative reconstruction algorithm..$^{29,30}$ Previously, we have shown that combining the SIM images using a linear reconstruction structured illumination (LR-SI) algorithm produces images with a flatter frequency response and better image fidelity at low signal intensities. $^{31}$

In this study, we combine LR-SI with DSLM. ${ }^{32}$ LR-SI has previously been combined with LSM to study early stage C. elegans embryos and other samples with linear dimensions of $\sim 50 \mu \mathrm{m}$ or less; ${ }^{14}$ here, we apply LR-SI to zebrafish larvae with a lateral dimension of $\sim 300 \mu \mathrm{m}$. Additionally, we combine digital scanning and SI with electronic CSD. ${ }^{33,34}$ We analyze LR-SI both with and without (LR-SI-non) the zero-order term and compare the performance to DSLM and RMS-SI. Despite requiring more intensive postprocessing, LR-SI-non achieves a final optically sectioned image with higher contrast, while using the same number of frames, than the RMS method. Therefore, there is no sacrifice in imaging speed. We evaluate the performance of our DSLM system comparing RMS-SI and LR-SI with different parameters, imaging fluorescent bead phantoms and thick biological samples (zebrafish larvae) and compare the contrast enhancement. We apply this approach to imaging neural activity in Pentylenetetrazol (PTZ) treated 7-day postfertilization (dpf) zebrafish larvae using a GCaMP indicator and demonstrate two-color imaging at 7 frames per second (fps).

\section{Materials and Methods}

\subsection{Optical Setup and Control System}

The system, shown in Fig. 1, is a modification of the OpenSPIM platform. ${ }^{33}$ The system described here was developed to image zebrafish larvae expressing the GCaMP5 calcium indicator and red fluorescent protein (RFP). We use two lasers, a 488-nm laser
(Coherent OBIS LX $50 \mathrm{~mW}$ ) and a 561-nm laser (Coherent OBIS LS $50 \mathrm{~mW}$ ) to sequentially excite the fluorescence. The lasers are combined by a dichroic mirror (Thorlabs DMLP505T) for multicolor imaging, and they copropagate into the sample. First, a telescope lens pair expands the laser beam by a factor of 2 . The beam is then directed onto a dual-axis galvo mirror scanner (Thorlabs GVSM002). After the galvo mirror scanner, the light passes through a $0.6 \times$ reducing telescope lens pair, which reimages the galvo onto the back pupil of the illumination objective lens (Olympus UMPLFLN10X/W $3.3 \mathrm{~mm}$ WD, $0.3 \mathrm{NA}$ ). The light forms a Gaussian beam with a $6.5-\mu \mathrm{m}$ FWHM waist at the focal plane of the objective, which is scanned to form a scanning light sheet.

The detection objective lens (Olympus, UMPLFLN20X/W, $3.3 \mathrm{~mm}$ WD 0.5NA) collects the fluorescent light, and the image formed by the tube lens (Olympus U-TLU $180 \mathrm{~mm}$ efl) is relayed by two $4 f$ systems onto the sCMOS camera (Hamamatsu Orca Flash 4.0v2). The final magnification of the system is either 26.67 or 33.3. The 26.67 magnification gives an effective pixel size of $244 \mathrm{~nm}$ and FoV of $500 \times 250 \mu \mathrm{m}^{2}$. The $33.3 \mathrm{mag}$ nification gives an effective pixel size of $195 \mathrm{~nm}$ and FoV of $399.4 \times 199.7 \mu \mathrm{m}^{2}$. A multibandpass filter (Semrock FF01$514 / 605 / 730-25$ ) is placed in front of the camera. The two relay systems serve to both increase the magnification and provide access to the back pupil plane for an adaptive optics (AO) system. The AO system is not implemented in this work and will be described in a forthcoming publication.

The sample holder is placed in a 4-D translation stage (Picard industries USB 4D-STAGE) so that the sample position can be adjusted relative to the illumination and detection unit. We use the stage translation approach to acquire 3-D data. The sample is moved along the $z$ axis of the detection objective lens and a series of cross-sectional images of the sample are captured. The galvo scanner is driven by a sawtooth voltage waveform using a Digilent analog shield (DAC) and Arduino microcontrollers.

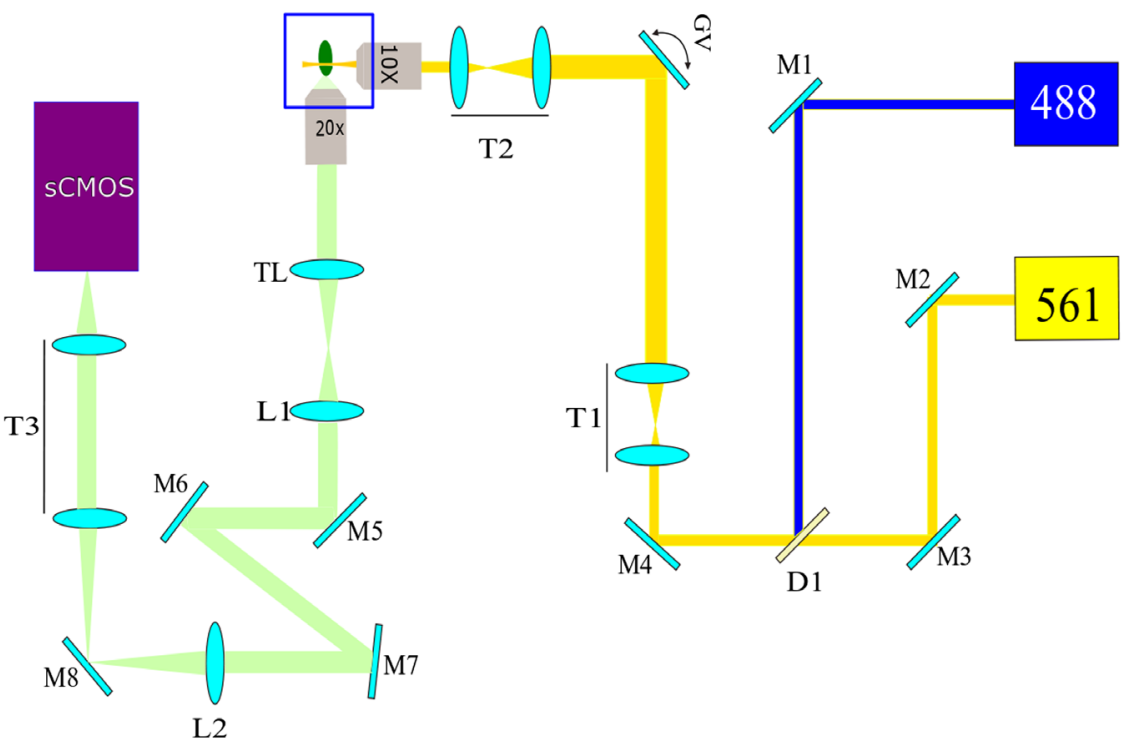

Fig. 1 Optical setup of the system. A 10 0.3 NA water dipping objective is used as the illumination objective lens and a $20 \times 0.5 \mathrm{NA}$ water dipping objective is used as the detection objective lens. M1-8: mirrors; D1, dichroic mirror; T1: 2x magnification lens pair (25 and $50 \mathrm{~mm}$ efl); T2 5/3 demagnfication lens pair ( 50 and $30 \mathrm{~mm}$ ); T3 2/3 demagnification lens pair ( $300 \mathrm{~m}$ and $200 \mathrm{~mm}$ efl); TL: tube lens $(180 \mathrm{~mm}$ efl); L1-2: relay lenses $(f 1=100 \mathrm{~mm} ; f 2=200 \mathrm{~mm})$; GV: two-axis galvo mirror. 

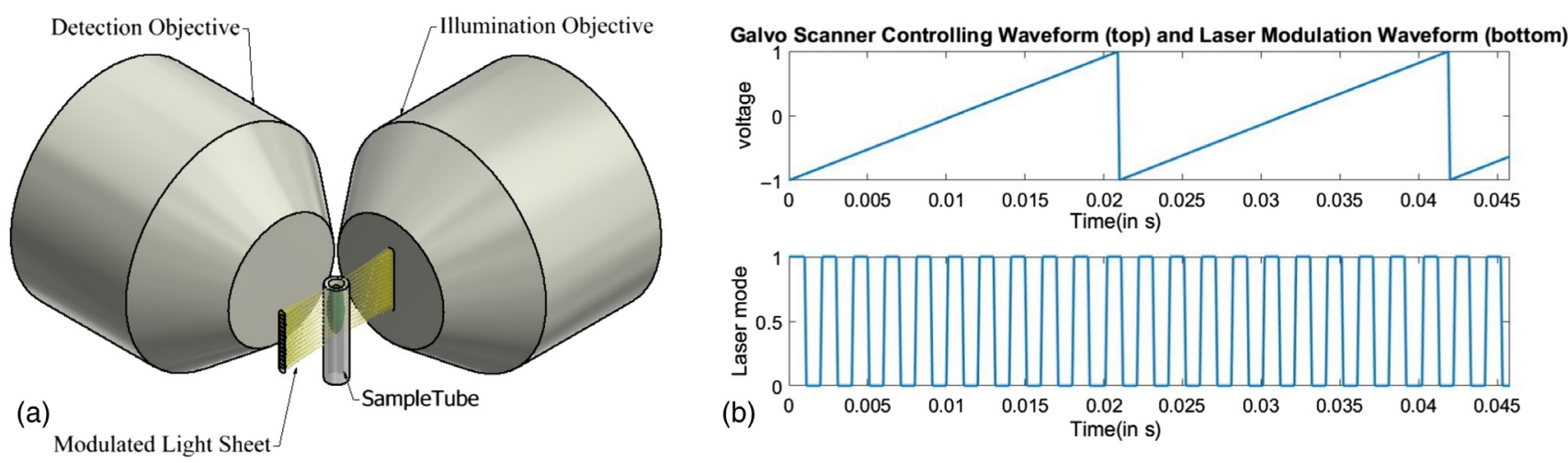

Fig. 2 Implementation of DSLM-SI. (a) Illustration of the SI light sheet configuration. (b) Top: controlling waveform of the galvo scanner for a $500 \mu \mathrm{m} \times 500 \mu \mathrm{m}$ FoV. Bottom: the laser modulation waveform with $k_{0}=0.0427 \mu \mathrm{m}^{-1}$ is shown.

We use the light sheet mode of the camera in which lines of pixels are read from the top of the sensor to the bottom. Figures 2(a) and 2(b) show the sawtooth waveform, which is used to drive the galvo scanner. This waveform is synchronized with the signal generated by the rolling shutter of the camera. The width of the rolling shutter is set to the full-width halfmaximum (FWHM) of the Gaussian beam in pixels. This way, only the activated area of the sensor is illuminated, and the background light is further decreased. The laser beams are alternately turned on for each vertical scan so that information from both channels is acquired in an interleaved manner. For high-speed, large FoV functional imaging, the exposure time for each pixel line is set to 0.76 and a $19 \mu$ s delay occurs between each line exposure. A delay of $0.73 \mathrm{~ms}$ between each vertical scan is also added. By using 1024 lines of pixels, we are able to achieve a 20.96-ms interval time between each frame, corresponding to 23.85 fps imaging speed for two channels. The system has a resolution of $510 \mathrm{~nm}$ laterally and $4.1 \mu \mathrm{m}$ axially in the green channel. For the purpose of enhancing the optical sectioning power of the system, we tested various sets of T1 lens pairs $(2 \times, 3 \times$, and $5 \times$ magnifications). This allows us to change the effective NA of the illumination objective lens, so that we can obtain a thinner sheet (FWHM: 7, 4, $2.8 \mu \mathrm{m}$ ). The microscope is controlled through a Dell precision 5810 Tower with 32 GB RAM, Intel(R)Xeon(R) E5-1603 v3 processor and customdeveloped LabVIEW program. The synchronization signal is achieved through two Arduino microcontroller boards and a chipKit u32 microcontroller board with a DAC shield.

\subsection{Structured Illumination and Image Reconstruction}

The SI pattern is formed by modulating the illumination laser beam. As the laser beam scans along the $y$ axis of the detection objective lens's focal plane, an Arduino microcontroller is used to digitally modulate the laser intensity to create the SI pattern. This approach allows us the flexibility to rapidly change the SI frequency so that the optimum optical sectioning power can be achieved. We acquire three images sequentially for each channel with a pattern phase difference of $2 \pi / 3$ between sequential images.

In linear fluorescence microscopy, the fluorescence intensity can be described as follows:

$$
I(\vec{r})=E(\vec{r}) \otimes H(\vec{r})
$$

where $E(\vec{r})$ is the fluorescence emission and $H(\vec{r})$ is the point spread function (PSF) of the system. $E(\vec{r})$ is the product of the sample labeling concentration $S(\vec{r})$, and the excitation intensity pattern of the system $L(\vec{r})$ :

$E(\vec{r})=S(\vec{r}) \cdot L(\vec{r})$

For SI, $L(\vec{r})$ is given by

$L(r)=L_{0}\left[1+\cos \left(\vec{k}_{0} \cdot \vec{r}+\varphi_{i}\right)\right]$

where $k_{0} \cong 2 \pi N A / \lambda$ is used for the optimal optical sectioning. The sinusoidal illumination results in superimposed shifted copies of the sample information $\tilde{D}_{ \pm k_{0}}(\bar{k})=\tilde{H}(\bar{k}) \tilde{S}\left(\bar{k} \mp \bar{k}_{0}\right)$ in addition to the WF image, where the tilde represents the Fourier transform.

After acquiring images of the sample, we employ both the RMS method ${ }^{25}$ and the LR-SI method ${ }^{31}$ to reconstruct the optically sectioned images. The RMS method is given by Eq. (4), the WF image by Eq. (5), and the LR-SI sectioned image by Eqs. (7) or (8):

$I_{\text {sectioned }}=\sqrt{\left(I_{\varphi_{1}}-I_{\varphi_{2}}\right)^{2}+\left(I_{\varphi_{2}}-I_{\varphi_{3}}\right)^{2}+\left(I_{\varphi_{1}}-I_{\varphi_{3}}\right)^{2}}$,

$I_{\mathrm{wf}}=\frac{1}{3}\left(I_{\varphi_{1}}+I_{\varphi_{2}}+I_{\varphi_{3}}\right)$.

In the LR-SI method, the shifted sample information, $\tilde{D}_{ \pm k_{0}}(\bar{k})$, can be separated with Eq. (6), in which $\varphi_{1}=0, \varphi_{2}=2 \pi / 3$, $\varphi_{3}=4 \pi / 3$, and $\mathcal{F}^{-1}\{\}$ represents the inverse Fourier transform:

$\left[\begin{array}{c}D_{0} \\ D_{+k_{0}} \\ D_{-k_{0}}\end{array}\right]=\frac{1}{3}\left[\begin{array}{ccc}1 & 1 & 1 \\ 2 e^{i \varphi_{1}} & 2 e^{i \varphi_{2}} & 2 e^{i \varphi_{3}} \\ 2 e^{-i \varphi_{1}} & 2 e^{-i \varphi_{2}} & 2 e^{-i \varphi_{3}}\end{array}\right]\left[\begin{array}{c}I_{\varphi_{1}} \\ I_{\varphi_{2}} \\ I_{\varphi_{3}}\end{array}\right]$, 


$$
I_{\mathrm{LR}-\mathrm{SI}}=\mathcal{F}^{-1}\left\{\frac{\left[\tilde{H}^{*}(\vec{k}) \tilde{D}_{0}(\vec{k})+\tilde{H}^{*}\left(\vec{k}+\vec{k}_{0}\right) \tilde{D}_{+k_{0}}\left(\vec{k}+\vec{k}_{0}\right)+\tilde{H}^{*}\left(\vec{k}-\vec{k}_{0}\right) \tilde{D}_{-k_{0}}\left(\vec{k}-\vec{k}_{0}\right)\right]}{\left[|\tilde{H}(\vec{k})|^{2}+\left|\tilde{H}\left(\vec{k}+\vec{k}_{0}\right)\right|^{2}+\left|\tilde{H}\left(\vec{k}-\vec{k}_{0}\right)\right|^{2}+w^{2}\right]}\right\} .
$$

The out-of-focus and scattering components of the signal are heavily concentrated near 0 spatial frequency. Therefore, to remove the scattering and background, we can calculate the LR-SI reconstruction without the zero-order term, Eq. (8). ${ }^{31}$ This method will be referred to as LR-SI-non:

$$
I_{\mathrm{LR}-\mathrm{SI}_{\text {no-zero }}}=\mathcal{F}^{-1}\left\{\frac{\left[\tilde{H}^{*}\left(\vec{k}+\vec{k}_{0}\right) \tilde{D}_{+k_{0}}\left(\vec{k}+\vec{k}_{0}\right)+\tilde{H}^{*}\left(\vec{k}-\vec{k}_{0}\right) \tilde{D}_{-k_{0}}\left(\vec{k}-\vec{k}_{0}\right)\right]}{\left[\left|\tilde{H}\left(\vec{k}+\vec{k}_{0}\right)\right|^{2}+\left|\tilde{H}\left(\vec{k}-\vec{k}_{0}\right)\right|^{2}+w^{2}\right]}\right\} .
$$

Similar approaches to linear removal of the out-of-focus light have been proposed by Heintzmann ${ }^{35}$ and Peng. ${ }^{36}$ In the approach by Heintzmann, implemented in a standard SIM, a Gaussian notch filter is used to remove the center of the OTF for the 0 and \pm 1 orders. Removing the center of the OTF for the \pm 1 orders is not necessary to remove out-of-focus light but could potentially aid in removing higher spatial frequency scattered light. We believe that this is unlikely to be a factor in our system. Peng implemented nonlinear SI in a two-photon Bessel light-sheet microscope. In this approach, numerical filters are used to combine the negative half of the negative orders with the positive half of the positive orders, also eliminating the zero order.

The parameter $w$ in Eqs. (7) and (8) is manually adjusted to achieve the optimal reconstructed image, which is evaluated by eye. The Wiener parameter is set to 0.31 for LR for all the data shown below. The reconstruction algorithm is written in Python with additional packages including Numpy and Scipy. As we mentioned above, the LR approach has a higher computational cost compared to the RMS method; the LR takes $315.3 \mathrm{~s}$ to process a $972 \mathrm{MB}$ image stack, which is 52.2 times longer than the RMS method.

\subsection{Sample Preparation}

In this work, we use dye phantoms, subdiffraction fluorescent beads and biological samples to test the performance of our system. For fluorescent dye phantom preparation, first, we mix the dye with DMSO into a $1.3-\mathrm{mM}$ stock solution, and then dilute the stock fluorescent dye (ThermoFisher Scientific Alexa Fluor 488 or Alexa Fluor 568) in $0.2 \%$ agar in the ratio of 1 to 500 . We then load the mixed solution into a FEP tube (Valco-TFEP130; OD $1.59 \mathrm{~mm}$, ID $0.76 \mathrm{~mm}$ ) for imaging. Both ends of the tube are plugged with $3 \%$ agar gel.

We prepared bead phantoms with different sizes of beads. We used 200-nm yellow-green (ThermoFisher Scientific F8811) fluorescent beads, 200-nm red fluorescent beads (ThermoFisher Scientific F8810) and 1- $\mu \mathrm{m}$ yellow-green fluorescent beads (ThermoFisher Scientific F8823). The beads are first diluted with deionized water $(18.2 \mathrm{M} \Omega / \mathrm{cm})$ in a ratio of $1: 1000$ separately as stock solutions. We mix the stock together with $0.2 \%$ agar gel in a ratio of 20 to 1 . Then, we inject the agar bead solution into an FEP tube and plug both ends of the tube with $3 \%$ agar gel.

\subsection{Zebrafish Studies}

Adult and larval zebrafish (Danio rerio) were obtained from lines maintained in the University of Georgia Zebrafish Facility following standard procedures. ${ }^{37}$ Embryos and larvae were staged using standard staging criteria. ${ }^{37,38}$ Wild-type fish of the WIK strain were originally obtained from the Zebrafish International Research Center (ZIRC). Fish transgenic for elavl3:GCaMP5G Tg/0 $^{\text {on a nacre (mitfa }}{ }^{w 2 /+}$ ) background were originally provided by Drs. M. Ahrens and D. Robson, ${ }^{7}$ and fish transgenic for gadlb:RFP were originally provided by Dr. S. Higashijima. ${ }^{39}$ All adult fish were maintained in an Aquatic Habitats (Apopka, Florida) multirack system. Habitat water consisted of reverse osmosis filtered/sterilized water to which sodium bicarbonate and other salts (Instant Ocean, Aquarium Systems, Inc., Mentor, Ohio) were added to maintain $\mathrm{pH}$ between 7.0 and 7.4 and conductivity between 400 and $430 \mu \mathrm{s}$. All experimental procedures were conducted in accordance with National Institutes of Health guidelines for use of zebrafish in research under protocols approved and overseen by the University of Georgia Institutional Animal Care and Use Committee.

For live zebrafish embryo sample preparation, we followed the protocol described in Ref. 40. First, the 7 dpf elavl3: GCaMP5g; gadlb:RFP; mitfa $a^{w 2 / w 2}$ transgenic larvae were paralyzed with alpha-bungarotoxin (125.25 $\mu \mathrm{M} \alpha$-BTX). For fish that were not already genetically mutated to experience abnormal neural events, $15 \mathrm{mM}$ PTZ was added to the environment to induce the abnormal neural condition. For fixed samples (structural imaging), samples were bathed in diluted tricane solution, which consists of $0.4 \%$ tricane-s solution that has been diluted with E3 media in a ratio of 0.042 . The fish were then placed in a $0.2 \%$ agarose solution mounted in the FEP tubing. Both ends were plugged with $3 \%$ agar gel.

\subsection{Data Analysis}

We calculated the signal-to-background ratio (SBR) and SNR for both the beads image and the sample image across all methods. For the beads images, we first calculated the background value using the mean intensity value of five different dark, featureless regions $(50 \times 50$ pixels $)$ around the image. The noise is calculated by taking the average of the standard deviations from the above five regions. The signal is calculated by taking the maximum intensity in each of five randomly selected regions of interest $(\mathrm{RoI})$, each $20 \times 20$ pixels, and then dividing by the background value to produce a ratio. For the biological sample images, we applied the same method. The difference was only that the RoIs were larger $(200 \times 200$ pixels $)$, and the backgrounds were also larger $(500 \times 500$ pixels $)$.

For the functional data analysis, Fig. 8(b), the intensity value of each time point is the sum of the RoI. The background value is established by averaging the intensity value over a 10 -s period and the noise is the standard deviation over the 10-s period. The SBR is calculated by taking the peak intensity over the 
background value, and the SNR is calculated using the peak intensity over the noise.

\section{Results and Discussion}

In LSFM, the thickness of the sheet is directly related to the optical sectioning performance of the system. A thinner beam will introduce much less out-of-focus light into the system and allow us to achieve a higher $z$ axis resolution. The thinner beam is also directly related to the modulation depth of the SI pattern because the ideal sectioning strength corresponds to a spatial frequency $k_{0}=\mathrm{NA} / \lambda$. For our system, the ideal SI pattern wavelength is $\sim 1 \mu \mathrm{m}$, smaller than the Gaussian beam waist. Therefore, a thinner beam will allow us to achieve much better contrast. Figure 3 shows different beam profiles for different amounts of initial magnification of the 488-nm laser and the pattern used for SI. The Rayleigh length of the beam is directly proportional to the beam waist $\left(Z_{R}=\pi w_{0}^{2} / \lambda\right)$, which means that the FoV is inversely proportional to optical sectioning strength.

We created beams with three different waists (BM1, BM2, and BM3) by expanding the laser beam by $2 \times, 3 \times$, and $5 \times$ using different lenses in the T1 lens pair in Fig. 1. BM1 has a FWHM of $6.60 \pm 0.18 \mu \mathrm{m}$ and a corresponding Rayleigh length of $305 \mu \mathrm{m}, \mathrm{BM} 2$ has a FWHM of $4.38 \pm 0.16 \mu \mathrm{m}$ with a Rayleigh length of $110 \mu \mathrm{m}$, and BM3 has a FWHM of $3.85 \pm 0.15 \mu \mathrm{m}$ with a Rayleigh length of $40 \mu \mathrm{m}$. For this work, BM1 is used, because it has the largest Rayleigh length and therefore results in the largest FoV, allowing us to capture the entire width of the zebrafish larval central nervous system.
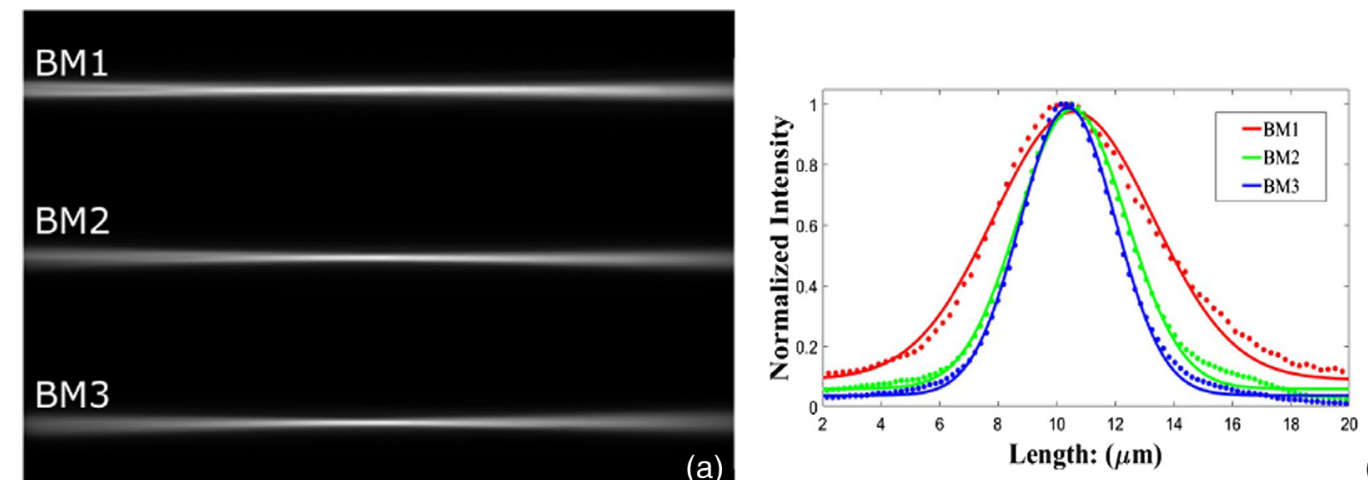

(b)
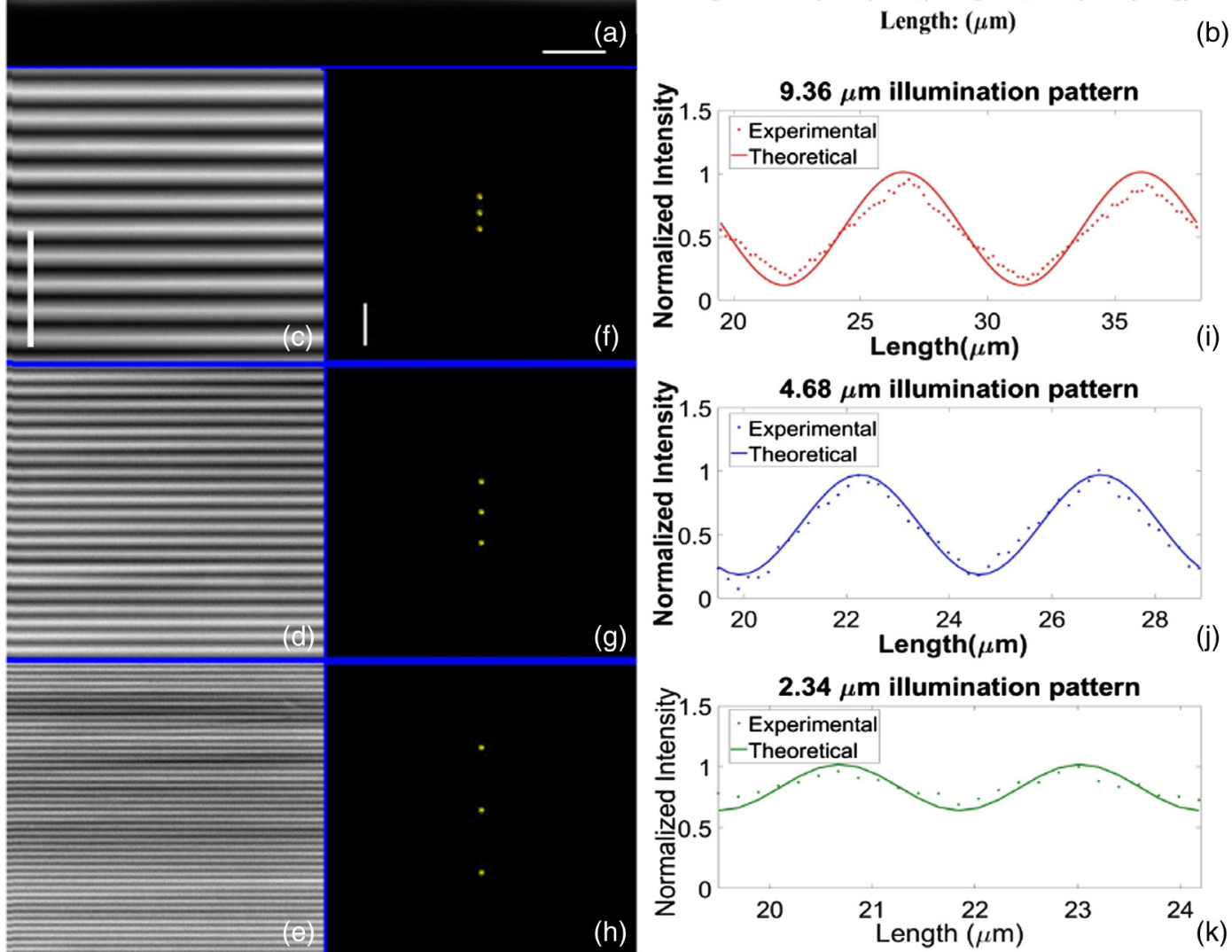

Fig. 3 Illumination beam profile and modulation depth of the SI pattern. Characterization using the dye phantom described in Sec. 2.4 (a). Beams BM1, BM2, BM3 are formed by using $2 \times, 3 \times, 5 \times$ magnification lens pair, respectively. The scale bar is $50 \mu \mathrm{m}$. (b) Intensity profile at the beam waist. (c)-(e) SI patterns generated using the $2 \times$ lens pair. SI patterns of $9.36,4.68$, and $2.34 \mu \mathrm{m}$. The FoV is $99.84 \times 99.84 \mu \mathrm{m}^{2}$, cutout from the $399.36 \times 399.36 \mu \mathrm{m}^{2}$ image. Scale bar is $40 \mu \mathrm{m}$. (f)-(h) Frequency space representations of (c)-(e), respectively. Scale bar is $0.3 \mu \mathrm{m}^{-1}$. (i)-(j) Normalized intensity plots of the cross-section of the images in (c) and (d). Dotted line is the experimental result and the solid line is the theoretical value. 
Figures 3(c)-3(e) demonstrate the different SI patterns created in the Alexa 561 dye phantom. Figures 3(f)-3(h) show the corresponding Fourier transforms to Figs. 3(c)-3(e). Figures 3(i)-3(k) show the theoretical modulation depth and the experimental results with the fluorescent dye phantom. Here, we used the 48-pixel pattern $(9.36 \mu \mathrm{m})$, which provides the best modulation depth with the $6.60-\mu \mathrm{m}$ beam waist, which is critical for later reconstruction.

Figure 4 compares the optical sectioning performance of the WF DSLM system to RMS-SI, LR-SI, and LR-SI-non methods using the 200-nm YG fluorescent bead phantom. The images are acquired with an illumination pattern wavelength of $9.36 \mu \mathrm{m}$. Figures $4(\mathrm{a})-4(\mathrm{c})$ are $99.84 \times 99.84 \mu \mathrm{m}^{2}$ regions from the $399.36 \times 399.36 \mu \mathrm{m}^{2}$ image. The PSF is measured using the intensity profile of the fluorescent beads. Ten beads are measured from different sets of images. The lateral PSF is plotted in Fig. 4(i), and Fig. 4(j) is the axial PSF. The WF image results in a FWHM of $631 \pm 19 \mathrm{~nm}$ in the $y$ axis and $4.49 \pm 0.25 \mu \mathrm{m}$ in the $z$ axis, while the FWHM of the PSF with RMS-SI is $623 \pm 23 \mathrm{~nm}$ and $4.48 \pm 0.28 \mu \mathrm{m}$. That of the LR-SI image is $478 \pm 19 \mathrm{~nm}$ and $4.01 \pm 0.23 \mu \mathrm{m}$, and that of the LR-SI-non is $470 \pm 15 \mathrm{~nm}$ and $3.86 \pm 0.29 \mu \mathrm{m}$. The resolution with LR-SI is better due to the Wiener filter in Eqs. (7) and (8), which boosts the higher frequencies. We calculated the SBR of each method; the LR-SI-non image shown in Fig. 4(d) has a SBR of $146.5 \pm$ 45.5, whereas the images in Figs. 4(a)-4(c) have SBRs of $6.6 \pm$ 1.25 (WF), $103.2 \pm 26.7$ (RMS-SI), and $13.87 \pm 3.25$ (LR-SI), respectively. LR-SI-non yields a SBR 23.8 times higher than that of the WF image and $42 \%$ higher than that of the widely adopted RMS-SI method. WF has a SNR of $368.9 \pm 75.1$.
The SNRs for RMS-SI, LR-SI, and LR-SI-non are $167.8 \pm 43.5$ $319.4 \pm 74.9$, and $181.0 \pm 56.2$.

Then, we tested our system with thick biological samples, zebrafish larvae, using a 11.7- $\mu \mathrm{m}$ illumination pattern with the $2 \times$ magnification lens pair. Figure 5 illustrates the gad $1 b: R F P$ expression in a 7-day old zebrafish larva's central nervous system, acquired in the DSLM-SI mode. Figures 5(a)-5(d), from left to right, are the WF, RMS-SI, LR-SI, and LR-SI-non images. Enlarged cutouts from Figs. 5(a)-5(d) are shown in Figs. 5(e)-5(h), and the contrast enhancement is demonstrated through the line intensity $y$-profile at the location noted in Fig. 5(e) for each of the methods. The line intensity profile is shown in Fig. 6. The LR-SI-non has better contrast in comparison with LR-SI, due to the removal of the zero-order term during the reconstruction process, which eliminates the scattering component. We then compared the SBR and SNR values across the four methods, LR-SI-non shows the highest SBR value, which is $194.8 \pm 26.7$, and WF, RMS, and LR-SI have values of $26.1 \pm 4.4,48.5 \pm 9.8$, and $31.8 \pm 4.4$, respectively. Unlike the result that we acquired using bead phantoms, the LR-SI has an SNR value of $335.5 \pm 46.7$; the SNR of LR-SInon is $254.6 \pm 34.9$; the SNR of RMS-SI is $71.8 \pm 14.6$; that of WF is $116.8 \pm 19.6$. LR-SI-non's SNR is 3.5 times higher than that of RMS-SI.

LR-SI-non and RMS-SI have the largest SBR values because they remove background-out-of-focus and scattered-light. LR-SI-non has higher SNR than RMS-SI because both the +1 and -1 SI orders contribute to the image while RMS-SI effectively isolates only one order. The relative values of SBR and SNR are different for beads and fish because the frequency
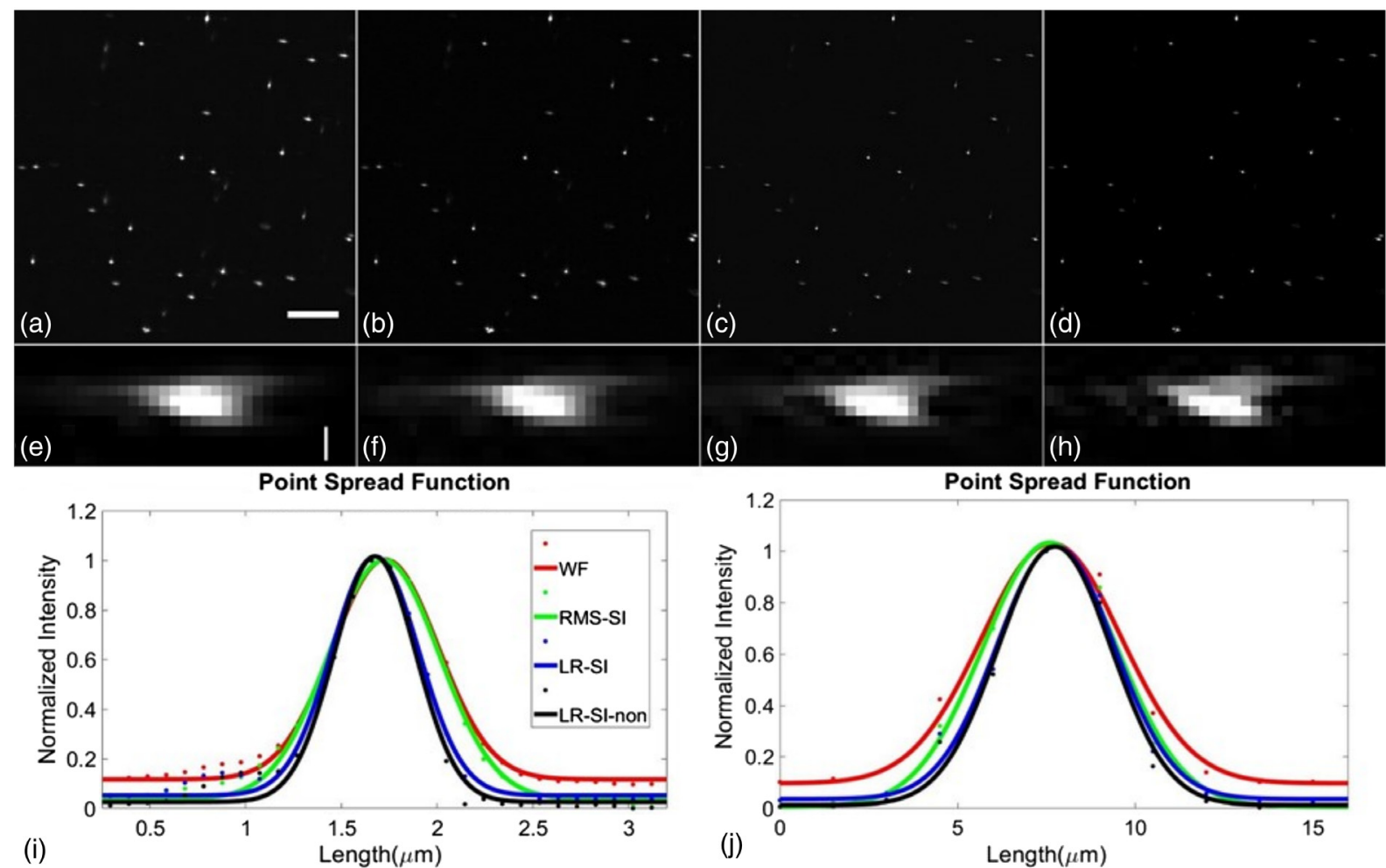

Fig. $4200 \mathrm{~nm}$ YG fluorescent bead phantom measured with different methods. (a) WF, (b) RMS-SI, (c) LR-SI, and (d) LR-SI-non. Image size $99.84 \times 99.84 \mu \mathrm{m}^{2}$ and scale bar is $20 \mu \mathrm{m}$. (e)-(h) Axial cross-sections of individual beads using the methods corresponding to (a)-(d). Axial step size is $1.5 \mu \mathrm{m}$. Image size $3.22 \times 18 \mu \mathrm{m}^{2}$ and scale bar is $5 \mu \mathrm{m}$. (i) Intensity profile of lateral PSF along the $y$ axis. (j) Intensity profile of axial PSF of the system. 


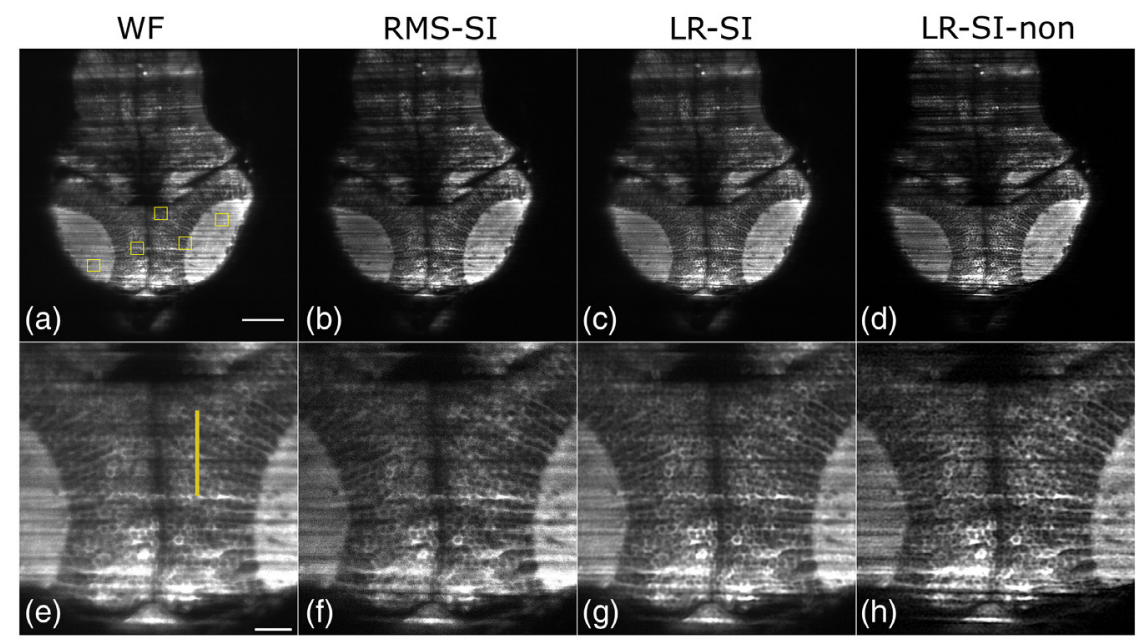

Fig. 5 Maximum intensity projections of 7-day-old elavl3:GCaMP5g; gad1b:RFP; mitfa ${ }^{w^{2} / w^{2}}$ zebrafish larva. (a)-(d) WF, RMS-SI, LR-SI, and LR-SI-non reconstructed images of the red channel of zebrafish larva's central nervous system, respectively. WF and RMS images are scaled up $2 \times$ bicubicly to match the pixel count of the LR-SI method. The image size is $500 \times 500 \mu \mathrm{m}^{2}$ and scale bar shown in (a) is $75 \mu \mathrm{m}$. (e)-(h) $183.4 \times 183.4 \mu \mathrm{m}^{2}$ cutouts from (a)-(d) and the scale bar shown in (e) is $25 \mu \mathrm{m}$. The yellow line indicates the position of the cross-sections in Fig. 6.

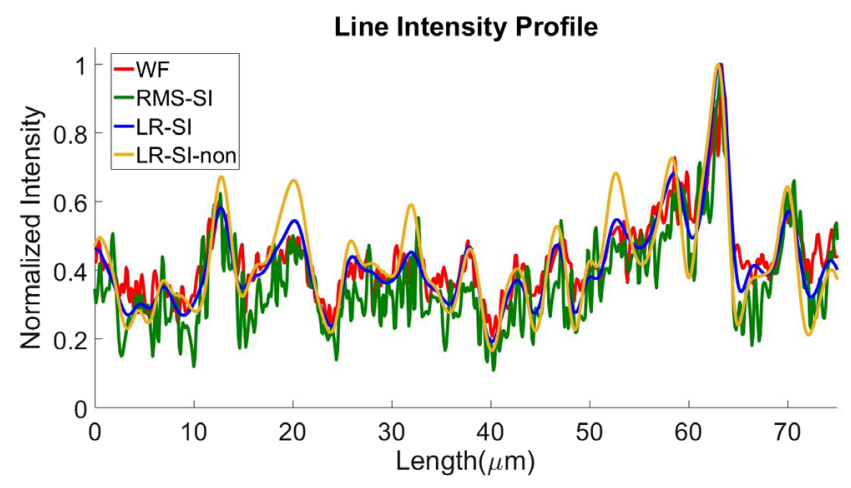

Fig. 6 Normalized intensity profiles along line at the location noted in Fig. 5(e) for each of the reconstruction methods. The Wiener parameter $(w)$ is set to 0.31 for the LR.

content of the image as well as the amount of background light in the image strongly affect how the LR-SI and LR-SI-non techniques compare to WF and RMS-SI. The images of zebrafish larvae have more low-frequency content compared to the bead phantoms. Different amounts of background light can be due to different amounts of scattering in the sample, and misalignment of the light sheet can also lead to more background light.

Figure 7 shows three different time points during a 2-min video clip, comparing the four different methods. From top to bottom, the series of images shows the WF, RMS-SI, LRSI, and LR-SI-non methods, respectively. From left to right, the interval between each time point is $0.5 \mathrm{~s}$. It shows that neural activity can be captured by our system with an effective twocolor rate of $7 \mathrm{fps}$.

The intensity change is plotted as a function of time in Fig. 8. LR-SI-non has a SBR of 2.8 and LR-SI has a SBR of 2.4, which shows that LR-SI-non has higher peak signals relative to the background. However, the LR-SI-non also results in lower SNR compared to LR-SI (91 compared to 350). In Fig. 8, neural events can be observed at $t \cong 0 \mathrm{~s}, t \cong 75 \mathrm{~s}$, and $t \cong 97 \mathrm{~s}$. The optic tectum of the larval zebrafish is a major part of the

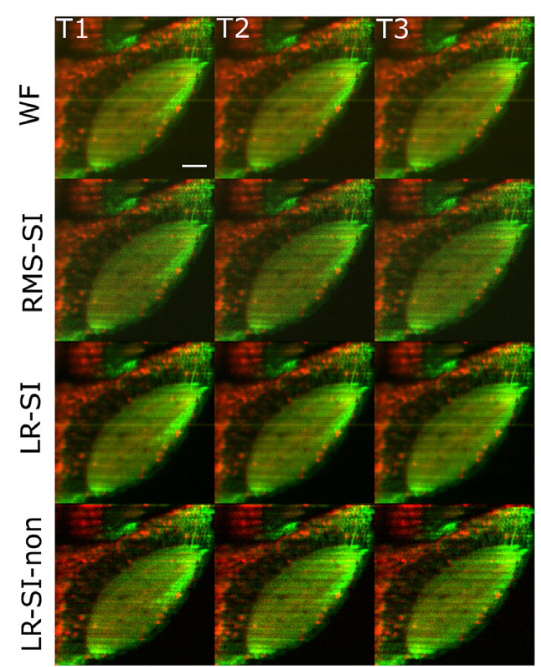

Fig. 7 Maximum intensity projection of 7-day old elavl3:GCaMP5g; gad1b:RFP; mitfa ${ }^{\text {w2/w2 }}$ zebrafish larva treated with 15-mM PTZ at three different time points. $195 \mu \mathrm{m} \times 195 \mu \mathrm{m}$ cutouts from $199.7 \times$ $399.4 \mu \mathrm{m}^{2}$ images are shown. The scale bar is $30 \mu \mathrm{m}$, and the images are acquired with an illumination pattern of $9.36 \mu \mathrm{m}$ at a speed of $\sim 48$ raw fps, corresponding to seven reconstructed two-color fps. The interval between each time point is $0.5 \mathrm{~s}$ (Video Collage 1, MPEG4, 5.58 MB [URL: https://doi.org/10.1117/1.NPh.6.1.015009.1]).

midbrain and consists of a large number of unmyelinated axons. This results in a relatively uniform fluorescent region in the image. This region is largely contained within the lower frequency bandwidth. Removal of the zero-order term during the reconstruction process results in a lower intensity of the signal, which leads to lower SNR in the RoI.

When imaging large samples, absorption and scattering structures inside the sample in the excitation light path cause stripe artifacts. ${ }^{41}$ Figure 9 shows 3 -D stacks at a range of depths as a comparison between different methods. Here, we observe that stripe artifacts become much more noticeable as we image deeper into the sample. This is due to the extra tissue, which 

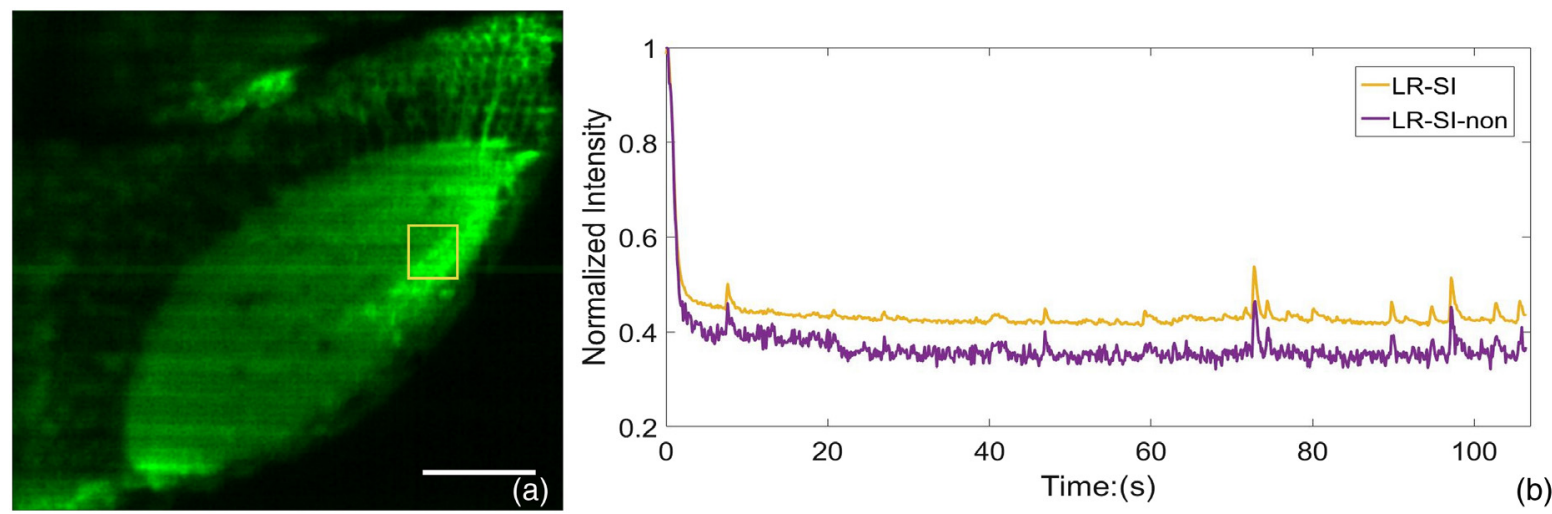

Fig. 8 LR-SI image of a 7-day-old elav/3:GCaMP5g; gad1b:RFP; mitfa ${ }^{\text {w2/w2 }}$ zebrafish larva treated with $15 \mathrm{mM}$ PTZ. (a) Integration of the intensity at the location of the Rol is calculated. (b) Intensity change of the Rol noted in (a) plotted as a function of time. LR-SI-non shows higher peak signals relative to the background compared to LR-SI. However, the LR-SI-non results in lower SNRs relative to LR-SI method. The scale bar is $40 \mu \mathrm{m}$.

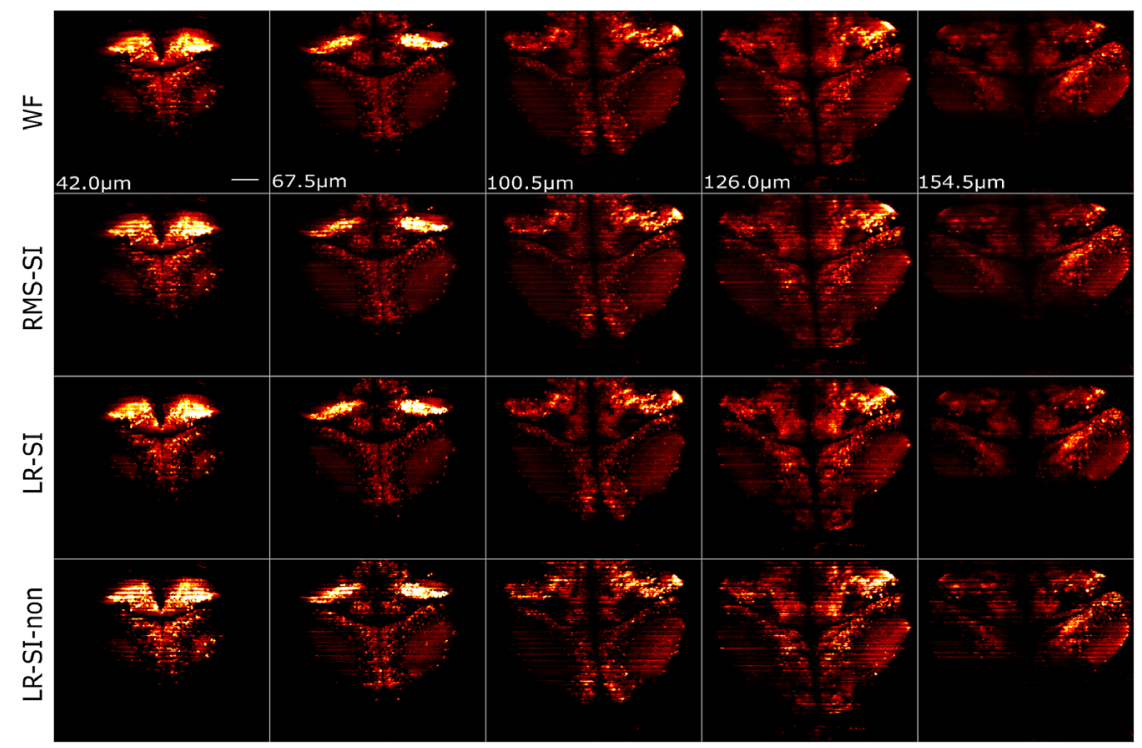

Fig. 9 Slices from a 3-D image stack of a 7-day-old elavl3:GCaMP5g; gad1b:RFP; mitfa ${ }^{\text {w2/w2 }}$ zebrafish larva with DSLM-SI. From the top to bottom row: WF, RMS-SI, LR-SI, and LR-SI-non. Each column is a different depth into the sample. image size is $399.36 \mu \mathrm{m} \times 399.36 \mu \mathrm{m}$ and the scale bar is $50 \mu \mathrm{m}$.

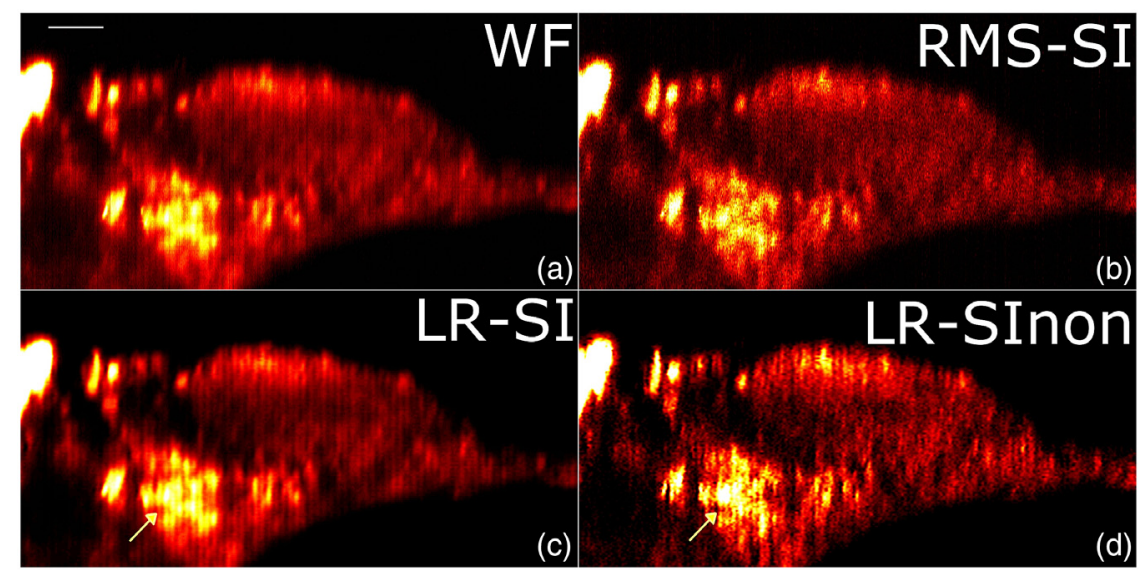

Fig. 10 Comparison of optical sectioning performance. $x-z$ planes from 3-D stack shown in Fig. 9: (a) WF, (b) RMS-SI, (c) LR-SI, and (d) LR-SI-non. Scale bar in (a) is $25 \mu \mathrm{m}$ and image size is $252 \mu \mathrm{m} \times 210 \mu \mathrm{m}$. 
introduces more scattered light. In addition, the stripe artifacts are much more obvious in RMS-SI and LR-SI-non, because the stripe artifacts are part of the high frequency structure of the image. ${ }^{42}$ Figure 10 shows axial cross-sections of the 3-D volume, illustrating the comparison between all four methods. LR-SI-non, Fig. 10(d), yields a stronger optically sectioned image. However, the stripe artifact is more apparent in comparison with Fig. 10(c). This is noted with a yellow arrow in Figs. 10(c) and 10(d). Various computational methods ${ }^{41,43}$ have been developed to mitigate the stripe artifacts, however, the large amount of data in LSFM images exponentially increases the computational cost of these methods. ${ }^{44}$ Multidirectional SPIM $^{42}$ is a promising method for alleviating stripe artifacts; however, it is not compatible with SI. Self-reconstructing beams have been shown to mitigate stripe artifacts and are compatible with DSLM-SI and, therefore, could be used in combination with DSLM-SI and LR-SI reconstruction. ${ }^{45,46}$ Recently, two approaches ${ }^{44,47}$ that are also compatible with DSLM-SI have been developed to address this issue. We are also working on an approach to mitigating stripe artifacts that is compatible with DSLM-SI.

The effect of optical aberrations on SI has been studied previously, and the aberrations have been shown to decrease pattern contrast and decrease the SNR of the reconstructed image. ${ }^{48,49}$ By using FEP tubing to mount our samples, we have minimized the effect of spherical aberration in our system as can be seen by the measured axial width of the PSF, which is in good agreement with the theoretically expected PSF width from a well-corrected $0.5 \mathrm{NA}$ objective $\left(2 \lambda / \mathrm{NA}^{2} \approx 4.0 \mu \mathrm{m}\right)$. Aberrations could be further controlled through the use of $\mathrm{AO} .^{50}$

\section{Conclusion}

In conclusion, we have demonstrated the combination of linear SI with LSFM to image neural activity in the central nervous system of $7 \mathrm{dpf}$ zebrafish larvae. Using linear SI, we can image 2-D planes in two colors at $7 \mathrm{fps}$. Linear SI shows higher SNR than both conventional DSLM and RMS-SI. In highly scattering sections, the scattering signal can be eliminated by removing the zero-order term in the linear SI reconstruction. Linear SI with the zero order removal also shows superior axial sectioning compared to RMS-SI in our setup, where the axial resolution of the imaging objective is comparable to the thickness of the light sheet. As the imaging plane is moved deeper into the sample where the sample is thicker, stripe artifacts become apparent due to absorption and scattering of the excitation beam. To address this problem, multiple computational approaches ${ }^{41,43,51}$ could be applied.

We are currently using the system to investigate neural activity in PTZ-treated zebrafish and different zebrafish mutants. Future work will involve making several improvements to the system including the use of fast axial scanning to enable volumetric imaging and the implementation of $\mathrm{AO}$ in the system to improve the resolution of the system.

\section{Disclosures}

No conflicts of interest, financial or otherwise, are declared by the authors.

\section{Acknowledgments}

This work was supported by the National Institutes of Health (Grant No. R01NS090645) and the National Science
Foundation (Grant No. 1350654). We thank Scott C. Baraban for useful discussions.

\section{References}

1. S. C. Baraban, "Emerging epilepsy models: insights from mice, flies, worms and fish," Curr. Opin. Neurol. 20(2), 164-168 (2007).

2. B. P. Grone and S. C. Baraban, "Animal models in epilepsy research: legacies and new directions," Nat. Neurosci. 18, 339-343 (2015).

3. J. Huisken et al., "Optical sectioning deep inside live embryos by selective plane illumination microscopy," Science 305, 1007-1009 (2004).

4. T. C. Fadero et al., "LITE microscopy: tilted light-sheet excitation of model organisms offers high resolution and low photobleaching," J. Cell Biol. 217, 1869-1882 (2018).

5. E. H. K. Stelzer and S. Lindek, "Fundamental reduction of the observation volume in far-field light microscopy by detection orthogonal to the illumination axis: confocal theta microscopy," Opt. Commun. 111(5), 536-547 (1994).

6. W. Denk, J. H. Strickler, and W. W. Webb, "Two-photon laser scanning fluorescence microscopy," Science 248(4951), 73-76 (1990).

7. M. B. Ahrens et al., "Whole-brain functional imaging at cellular resolution using light-sheet microscopy," Nat. Methods 10, 413-420 (2013).

8. T. Panier et al., "Fast functional imaging of multiple brain regions in intact zebrafish larvae using selective plane illumination microscopy," Front. Neural Circuits 7, 65 (2013).

9. F. O. Fahrbach et al., "Rapid 3D light-sheet microscopy with a tunable lens," Opt. Express 21(18), 21010-21026 (2013).

10. Y. Liu et al., "Imaging a seizure model in zebrafish with structured illumination light sheet microscopy," Proc. SPIE 10499, 104991C (2018).

11. A.-K. Gustavsson et al., "3D single-molecule super-resolution microscopy with a tilted light sheet," Nat. Commun. 9(1), 123 (2018).

12. M. B. M. Meddens et al., "Single objective light-sheet microscopy for high-speed whole-cell 3D super-resolution," Biomed. Opt. Express 7(6), 2219-2236 (2016).

13. P. Hoyer et al., "Breaking the diffraction limit of light-sheet fluorescence microscopy by RESOLFT," Proc. Natl. Acad. Sci. U.S.A. 113(13), 3442-3446 (2016).

14. L. Gao et al., "Noninvasive imaging beyond the diffraction limit of 3D dynamics in thickly fluorescent specimens," Cell 151(6), 1370-1385 (2012).

15. R. Tomer et al., "Quantitative high-speed imaging of entire developing embryos with simultaneous multiview light-sheet microscopy," Nat. Methods 9, 755-763 (2012).

16. O. E. Olarte et al., "Decoupled illumination detection in light sheet microscopy for fast volumetric imaging," Optica 2(8), 702-705 (2015).

17. H.-U. Dodt et al., "Ultramicroscopy: three-dimensional visualization of neuronal networks in the whole mouse brain," Nat. Methods 4, 331-336 (2007).

18. A. K. Glaser et al., "Light-sheet microscopy for slide-free non-destructive pathology of large clinical specimens," Nat. Biomed. Eng. 1, 0084 (2017).

19. F. O. Fahrbach et al., "Light-sheet microscopy in thick media using scanned Bessel beams and two-photon fluorescence excitation," Opt. Express 21(11), 13824-13839 (2013).

20. T. A. Planchon et al., "Rapid three-dimensional isotropic imaging of living cells using Bessel beam plane illumination," Nat. Methods 8, 417-423 (2011).

21. F. O. Fahrbach and A. Rohrbach, "A line scanned light-sheet microscope with phase shaped self-reconstructing beams," Opt. Express 18(23), 24229-24244 (2010).

22. T. Vettenburg et al., "Light-sheet microscopy using an Airy beam," Nat. Methods 11, 541-544 (2014).

23. Z. Yang et al., "A compact Airy beam light sheet microscope with a tilted cylindrical lens," Biomed. Opt. Express 5(10), 3434-3442 (2014).

24. R. M. Power and J. Huisken, "A guide to light-sheet fluorescence microscopy for multiscale imaging," Nat. Methods 14, 360-373 (2017). 
25. M. A. A. Neil, R. Juskaitis, and T. Wilson, "Method of obtaining optical sectioning by using structured light in a conventional microscope," Opt. Lett. 22(24), 1905-1907 (1997).

26. T. Breuninger, K. Greger, and E. H. K. Stelzer, "Lateral modulation boosts image quality in single plane illumination fluorescence microscopy," Opt. Lett. 32(13), 1938-1940 (2007).

27. P. J. Keller et al., "Fast, high-contrast imaging of animal development with scanned light sheet-based structured-illumination microscopy," Nat. Methods 7(8), 637-642 (2010).

28. B. Hu, D. Bolus, and J. Q. Brown, "Improved contrast in inverted selective plane illumination microscopy of thick tissues using confocal detection and structured illumination," Biomed. Opt. Express 8(12), 5546-5559 (2017).

29. J. Mertz and J. Kim, "Scanning light-sheet microscopy in the whole mouse brain with HiLo background rejection," J. Biomed. Opt. 15(1), 016027 (2010).

30. T. J. Schröter et al., "Scanning thin-sheet laser imaging microscopy (sTSLIM) with structured illumination and HiLo background rejection," Biomed. Opt. Express 3(1), 170-177 (2012).

31. B. Thomas, M. Momany, and P. Kner, "Optical sectioning structured illumination microscopy with enhanced sensitivity," J. Opt. 15(9), 094004 (2013).

32. P. J. Keller et al., "Reconstruction of zebrafish early embryonic development by scanned light sheet microscopy," Science 322, 1065-1069 (2008).

33. P. G. Pitrone et al., "OpenSPIM: an open-access light-sheet microscopy platform," Nat. Methods 10(7), 598-599 (2013).

34. L. Silvestri et al., "Confocal light sheet microscopy: micron-scale neuroanatomy of the entire mouse brain," Opt. Express 20(18), 20582-20598 (2012).

35. K. Wicker et al., "Phase optimisation for structured illumination microscopy," Opt. Express 21(2), 2032-2049 (2013).

36. M. Zhao et al., "Cellular imaging of deep organ using two-photon Bessel light-sheet nonlinear structured illumination microscopy," Biomed. Opt. Express 5(5), 1296-1308 (2014).

37. M. Westerfield, A Guide for the Laboratory Use of Zebrafish (Danio rerio), University of Oregon Press, Eugene (2007).

38. C. B. Kimmel et al., "Stages of embryonic development of the zebrafish," Dev. Dyn. 203(3), 253-310 (1995).

39. C. Satou et al., "Transgenic tools to characterize neuronal properties of discrete populations of zebrafish neurons," Development 140(18), 3927-3931 (2013).

40. M. Weber, M. Mickoleit, and J. Huisken, "Multilayer mounting for long-term light sheet microscopy of zebrafish," J. Visualized Exp. (84), e51119 (2014)
41. X. Liang et al., "Stripe artifact elimination based on nonsubsampled contourlet transform for light sheet fluorescence microscopy," J. Biomed. Opt. 21(10), 106005 (2016).

42. J. Huisken and D. Y. R. Stainier, "Even fluorescence excitation by multidirectional selective plane illumination microscopy (mSPIM)," Opt. Lett. 32(17), 2608-2610 (2007).

43. J. Fehrenbach, P. Weiss, and C. Lorenzo, "Variational algorithms to remove stationary noise: applications to microscopy imaging," IEEE Trans. Image Process. 21(10), 4420-4430 (2012).

44. A. K. Glaser et al., "Multidirectional digital scanned light-sheet microscopy enables uniform fluorescence excitation and contrast-enhanced imaging," Sci. Rep. 8(1), 13878 (2018).

45. M. C. Müllenbroich et al., "Bessel beam illumination reduces random and systematic errors in quantitative functional studies using light-sheet microscopy," Front. Cell. Neurosci. 12, 315 (2018).

46. F. O. Fahrbach, P. Simon, and A. Rohrbach, "Microscopy with selfreconstructing beams," Nat. Photonics 4, 780-785 (2010).

47. M. A. Taylor et al., "Diffuse light-sheet microscopy for stripe-free calcium imaging of neural populations," J. Biophotonics 11(12), e201800088 (2018).

48. B. Thomas et al., "Enhanced resolution through thick tissue with structured illumination and adaptive optics," J. Biomed. Opt. 20(2), 026006 (2015).

49. C. Preza, "Simulating structured-illumination microscopy in the presence of spherical aberrations," Proc. SPIE 7904, 79040D (2011).

50. C. Bourgenot et al., "3D adaptive optics in a light sheet microscope," Opt. Express 20(12), 13252-13261 (2012).

51. B. Münch et al., "Stripe and ring artifact removal with combined wavelet-Fourier filtering," Opt. Express 17(10), 8567-8591 (2009).

Yang Liu received his BS degree in biomedical engineering from the University of Georgia in 2015. Currently, he is a graduate student in the College of Engineering at the University of Georgia.

Peter Kner received his $\mathrm{PhD}$ in physics from the University of California Berkeley in 1998 in the field of ultrafast spectroscopy, working with Daniel Chemla. From 2004 through 2008, he worked at the University of California, San Francisco, in the groups of John Sedat and Mats Gustafsson. He joined the College of Engineering at the University of Georgia in 2009 where he is currently an associate professor.

Biographies of the other authors are not available. 